\title{
Effect of Inlet Clearance Gap on the Performance of an Industrial Centrifugal Blower with Parallel Wall Volute
}

\author{
Chinnasamy Hariharan and Mukka Govardhan \\ Thermal Turbomachines Laboratory, Department of Mechanical Engineering, \\ Indian Institute of Technology Madras, India \\ hari_pblr@yahoo.co.in,gova@iitm.ac.in
}

\begin{abstract}
While performing numerical simulations, it is general industrial practice to neglect the clearance gap between the impeller and the inlet duct. In the present work, the effect of clearance gap on the performance of an industrial sized centrifugal blower is simulated for two volutes of width ratios and various flow coefficients. The results show that the clearance has a positive effect at low mass flow rates. This is observed in the pressure rise (1.3\%) as well as in efficiency $(0.7 \%)$. At higher mass flow rates, it has a negative effect with a drop in efficiency of $1 \%$ and pressure drop of about $1.4 \%$. The effect of clearance gap on volute with higher width ratio is smaller when compared with the volute with smaller width ratio.
\end{abstract}

Keywords: Parallel wall volute, clearance gap, centrifugal blower.

\section{Introduction}

Centrifugal blowers have a wide range of applications, spanning from thermal power plants to chemical industries. Due to the cost effectiveness and low pressure ratios, the centrifugal fan with parallel volute is most preferred type of fan. In numerical simulations, some simplifications are made in the physics and geometry of the problem, so that the complexity of the problem is reduced. One such assumption is that there is no leakage of flow in the inlet region of centrifugal fans. This assumption is made in order to reduce the meshing complexity. This assumption is valid for most of the situations as the amount of clearance is only 0.5 to $2 \%$ of impeller outer diameter. However in the case of the centrifugal blower with parallel wall volute, the leakage flow has a significant effect on the performance of the blower. Lee [1] numerically simulated the effect of the clearance gap in the doublewidth double-inlet impeller and a double-discharge volute. In this study, he has shown that the effect of clearance can change the performance by as much as 5\%. He has also mentioned that the impact of the gap on the impeller performance is much less compared to the volute performance. In practice, most of the industrial blowers used in the thermal power plants have a typical impeller inlet diameter of $0.6 \mathrm{~m}-1 \mathrm{~m}$. For manufacturing purposes, a minimum clearance of 2 to $3 \mathrm{~mm}$ is usually maintained between the impeller and the inlet duct.

\section{Design}

In the present study, an industrial blower with a design speed and flow rate of $3000 \mathrm{rpm}$ and $24 \mathrm{~m}^{3} / \mathrm{s} \mathrm{respectively} \mathrm{is} \mathrm{chosen.}$ The specific work is $24000 \mathrm{~m}^{2} / \mathrm{s}^{2}$ and operating range is $12 \mathrm{~m}^{3} / \mathrm{s}$ to $26 \mathrm{~m}^{3} / \mathrm{s}$. The suction duct diameter as well as the impeller inlet diameter is maintained at $0.7 \mathrm{~m}$. The clearance between the suction duct and the impeller is maintained at $2 \mathrm{~mm}(0.6 \%$ of the inlet area) and the impeller consists of 15 circular arc blades with inlet and exit blade angle of $31^{\circ}$ and $48^{\circ}$. Volute is designed based on constant angular momentum principle. Parallel wall volutes with Width ratios of 4 and 5 (the ratio between the impeller exit width and the volute width) are used. The clearance is maintained constant for both types of volutes. Most industries use the volute with a higher width ratio for cost effectiveness. The impact of clearance when the width ratio changes is ascertained in the present investigations. As shown in Fig. 1 entire impeller and the suction duct are placed inside the volute. Hence clearance flow enters directly from the volute into the impeller. The suction duct extends into the impeller by $3 \mathrm{~mm}$. 


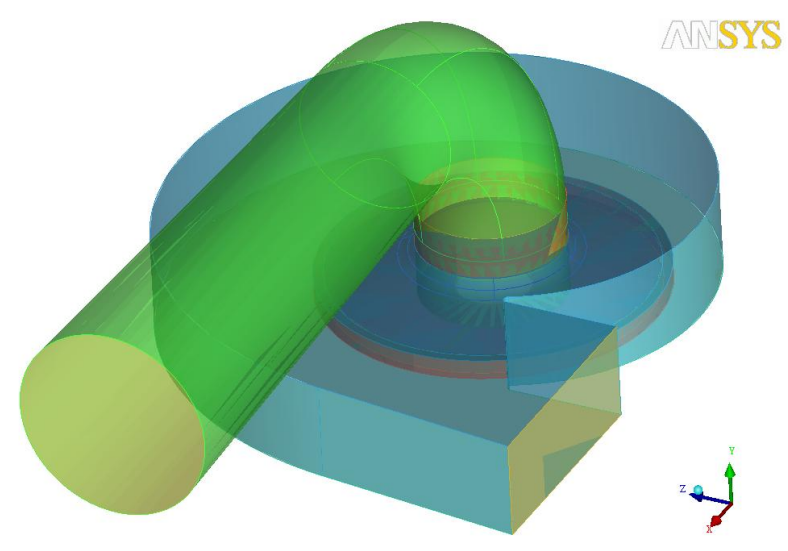

Fig. 1 Fan Assembly with R5 volute

\section{Computational methods}

Impeller is a rotating domain while the volute and the suction duct are stationary domains. The flow inside the volute is fully three dimensional, (Aayder et al. [2]). For numerical simulation a three dimensional domain is modeled. For impeller, the flow at exit of the impeller is not uniform along the circumference of the volute, (Braembussche et al. [3]). The impeller blades experiences different loading along the circumferential position. The volute casing is a non-axisymmetric body. All of the above reasons necessitated modeling of the entire fan. Numerical simulation is done using commercial CFD code CFX 14 using which the three dimensional mass, momentum and energy equations are solved. The high speed rotating turbulence flow inside the volute is captured using K- $\varepsilon$, turbulence model, Fahua [4].

In impeller, the equations are solved using a rotating frame with rotational speed of $3000 \mathrm{rpm}$, and the position of impeller is fixed with respect to the stationary components. The volute and suction duct variables are solved using the stationary frame. The transfer of information between the stationary and rotating domain is carried out using the Frozen Rotor Technique. The boundary conditions are total pressure at inlet equal to atmosphere; and at the outlet, mass flow is prescribed. The walls are smooth and adiabatic and the total energy is maintained constant.

As the blower operates at $\mathrm{M} \sim 0.5$, the flow is assumed compressible. The temperature at inlet is $300^{\circ} \mathrm{K}$. To simulate the flow without clearance cases, the clearance gap between the suction duct and impeller is defined as wall. The reliability and accuracy of solution is mainly dependent on boundary condition and meshing. The higher number of elements will give better accuracy requiring higher computational resources. After grid independence study, 5.5 million nodes in impeller, 4.5 million nodes in volute and 0.8 million nodes in suction duct were found to be adequate for reasonable results. The full hexahedral meshing is done using commercial mesh generation software ANSYS ICEM-CFD. The simulation is done using the k- $\mathcal{E}$ turbulence model and the $\mathrm{Y}+$ value was maintained less than 50. To reduce numerical diffusion, the mesh volume expansion factor was controlled so that it is less than 25 . To reduce the interface loss, the area ratio in the interface was maintained to be almost equal to 1 . This was done by providing proper number of nodes in radial, circumferential directions as well as an equal number of nodes on each side. The clearance gap is very small compared to the full domain, as shown in [Fig.2] Hence in order to capture the effect of clearance at the interface, $10 \times 1100$ nodes are placed in the clearance gap.

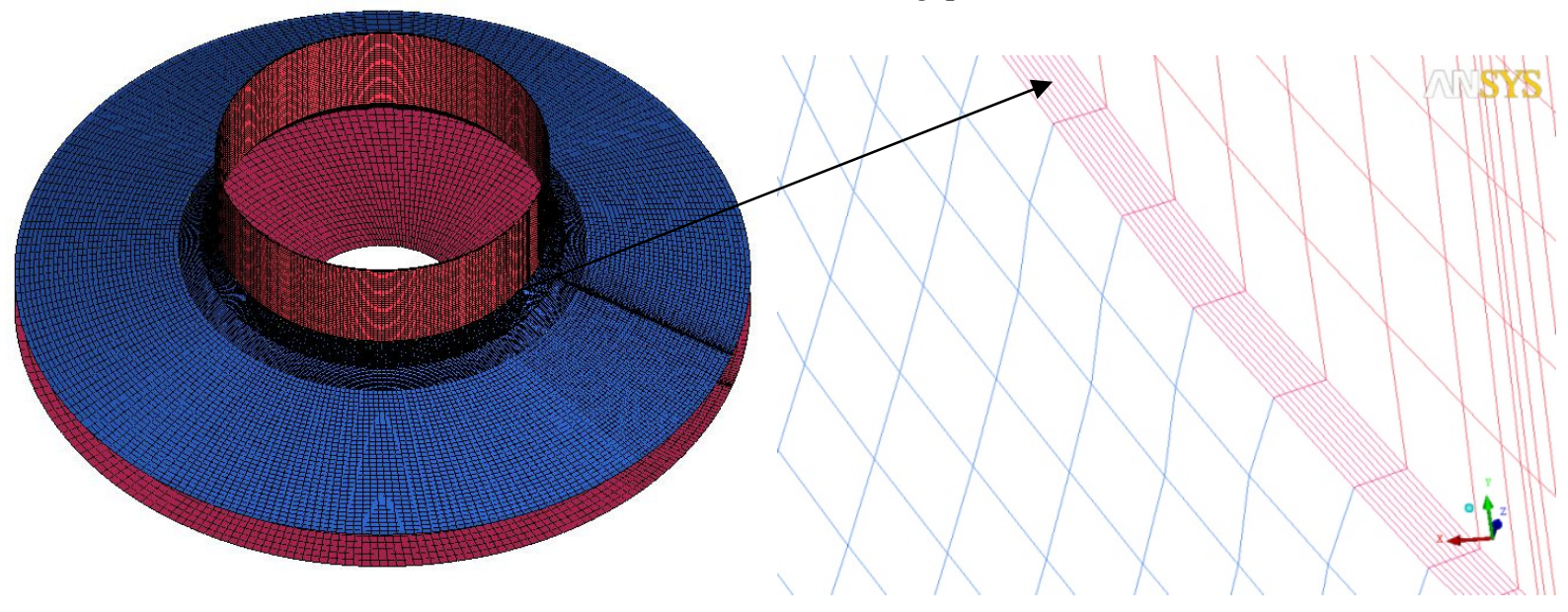

(a)

(b)

Fig. 2 (a) Impeller suction duct assembly inside the Volute

(b) Impeller suction duct assembly inside the Volute

The simulation is done for various volume flow rates (12 to $\left.26 \mathrm{~m}^{3} / \mathrm{s}\right)$ and width ratios of 4 and 5 . The inlet clearance has an effect on both the volute and the impeller aerodynamics, but the effect on volute is more compared to that on impeller Lee [1]. One of the main parameters deciding the clearance gap is the shroud shape. The effect of smooth connectivity between the 
suction duct and the impeller is investigated by Xiaomin Liu et al. [5]. He explained the importance of the shroud shape and how it can affect the performance of the blower by 2 to 3\%. In the impeller, the high energy (momentum) return flow mixes with the low energy inlet flow (entering the impeller from suction duct) and consequently produces a cross flow along the span of the blade. This creates an incidence loss even at the design flow rate and this effect increases during the off-design operation of the impeller, especially in the low mass flow regime. In addition to the flow coming from the suction duct, there is also a small disturbance because of a cross flow already existing at the $90^{\circ}$ turn which is present from the inner curve to the outer curvature side Kim et al. [6].

\section{Result and Discussion}

Turbomachine is an interesting mechanical system. It will always try to adjust its operating condition; a small change in the inlet condition to the impeller will affect the entire system performance. This change in the performance has been discussed both at the system as well as the component level.

\subsection{Stage Performance}

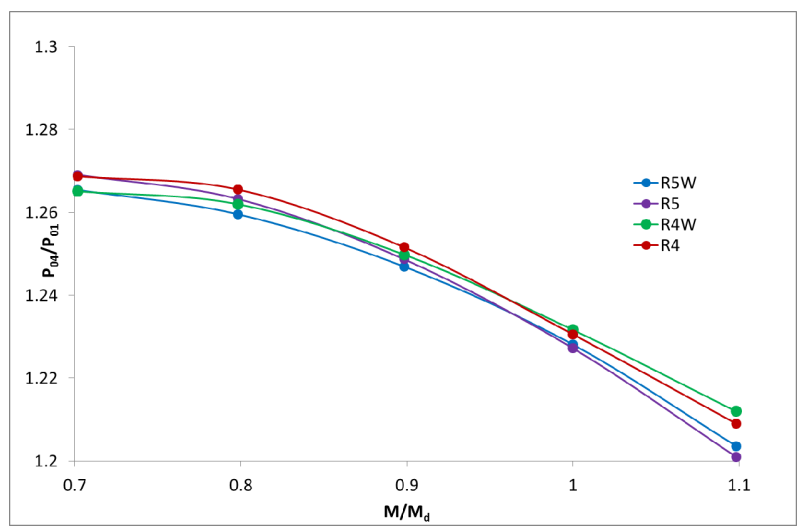

Fig. 4 Pressure ratio of fan for different mass flow rates

The total to total pressure ratio across the fan is plotted in Fig. 4. In the plot, R4 and R5 represents the simulations for volute width ratio $=4$ and volute width ratio $=5$ respectively; $\mathrm{R} 4 \mathrm{~W}$ and $\mathrm{R} 5 \mathrm{~W}$ represents the simulations without clearance for volute width ratio $=4$ and volute width ratio $=5$ respectively. Clearance gap produces higher pressure in low mass regime and lower pressure ratio at higher flow rates. However the magnitude is small compared to the total pressure developed by the machine $(+300 \mathrm{~Pa},-300 \mathrm{pa})$. In lower mass flow rates, the effect of the clearance is same for both R4 and R5 volute ratios. At higher mass flow rates the effect generated by the clearance gap in each of the volute ratios is different. The difference in the total pressures generated for with and without clearance cases is more for R4 than R5. The amount of fluid handled by the impeller in when the clearance gap is present is more compared to the case of without clearance. The return flow through the clearance from the volute to the impeller is always maintained at $0.31 \mathrm{~kg} / \mathrm{m}^{3}$ irrespective of the inlet mass flow rates or volute ratios (R4 or R5).

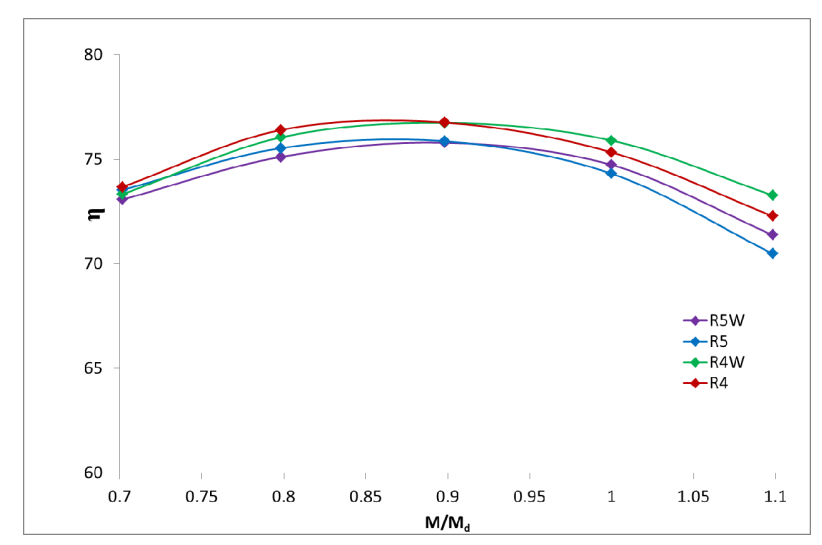

Fig. 5 Isentropic efficiency of fan for different mass flow rates

The total to total efficiency as a function of the flow rate is plotted in Fig. 5 The trend followed by the efficiency is similar to that of the pressure rise. Even though in lower mass flow regime the change in pressure rise between with clearance and without clearance cases is more compared to higher mass flow rates, the change in efficiency does not mirror this. The efficiency change in the lower mass flow rates is $-0.68 \%$ whereas at highest mass flow rate change is $+1 \%$. 


\subsection{Impeller Performance}

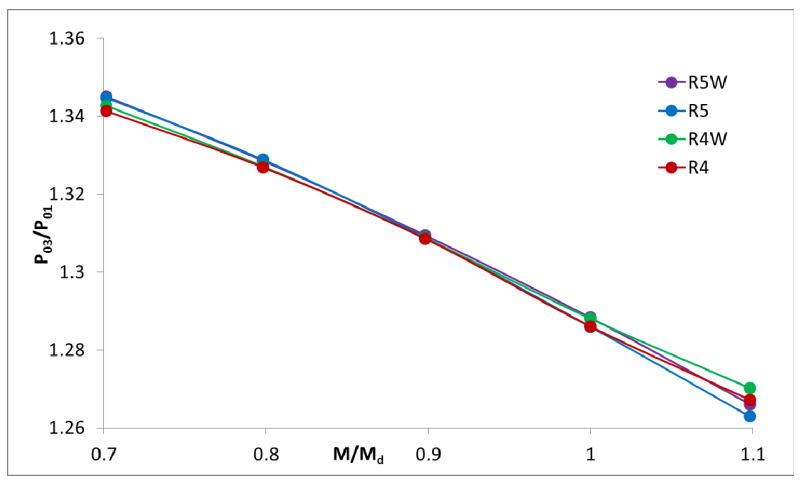

Fig. 6 Pressure ratio of impeller for different mass flow rates

In Fig. 6, the ratio between the impeller outlet and inlet total pressures is plotted. From figure, it can seen that without clearance the impeller is producing higher pressure rise at all mass flow rates. The aforementioned result is at the impeller level whereas at the system level, the effect of clearance at lower mass flow rates is positive whereas at higher mass flow rates, it is negative. For without clearance case, flow separation is more due to the lower energy of the flow entering the impeller and encountering a higher curved shroud. This causes a loss of energy. When the clearance is present, high energy jet flow coming from clearance gap reduces the separation in impeller inlet but causes an increase in the span wise disturbance and hence can lead to flow separation in the blade edge. The difference is minimum near the design mass flow conditions. The difference increases at both low and high mass flow rates. The slope of the difference is more at higher mass flow rate conditions when compared to the low mass flow rates.

The slope of difference in pressure rise is high in R4 volute when compared to the R5 volute. The magnitude remains the same in higher mass flow rates. But in lower mass flow rates the difference is much smaller in R4 compared to R5 (-130Pa, 30Pa).

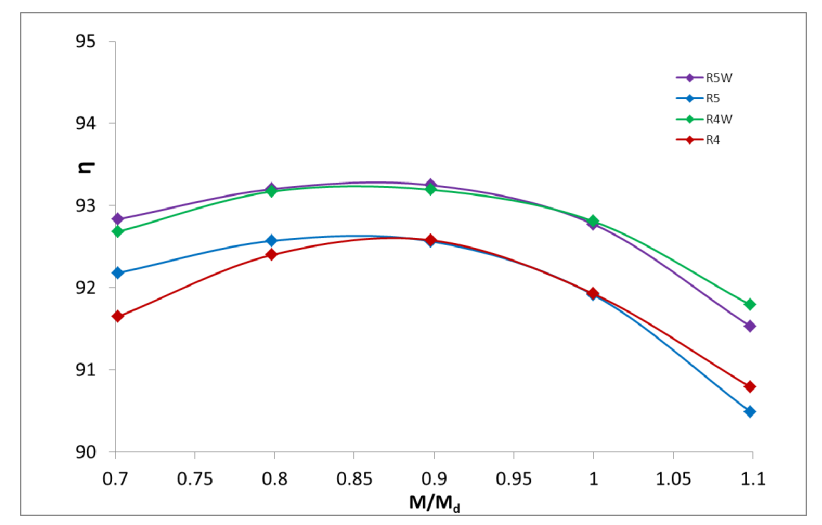

Fig. 7 Isentropic efficiency of impeller for different mass flow rates

The total to total efficiency of impeller is plotted in Fig. 7. The efficiency when the clearance is zero is more for all mass flow rates. Similar to the pressure rise, the difference is minimum, close to the design mass flow rates. However, it increases for both high and low mass flow rates.

In R4 and R5 volutes the magnitude of difference at the highest mass flow rate is $1 \%$ but at the lowest mass flow rate R4 has $1 \%$ as opposed to R5 which has only $0.68 \%$. The effect of clearance is more in R4 volute when compared to the R5 volute fan.

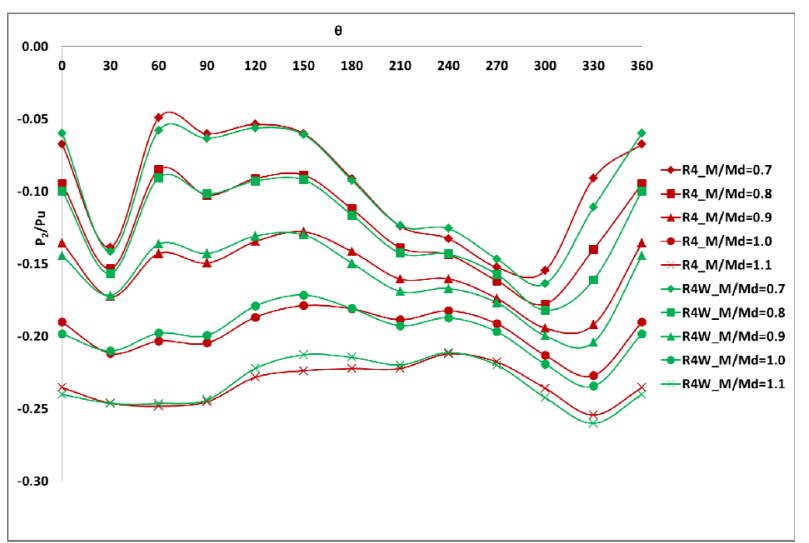

Fig. 8 Static pressure variation at inlet to impeller for R4 volute 
One of the main impacts of the clearance is the change in static pressure at inlet of the impeller. The static pressure at angular position is plotted in Fig.9. The volute tongue position is taken as the reference point $0^{\circ}$.

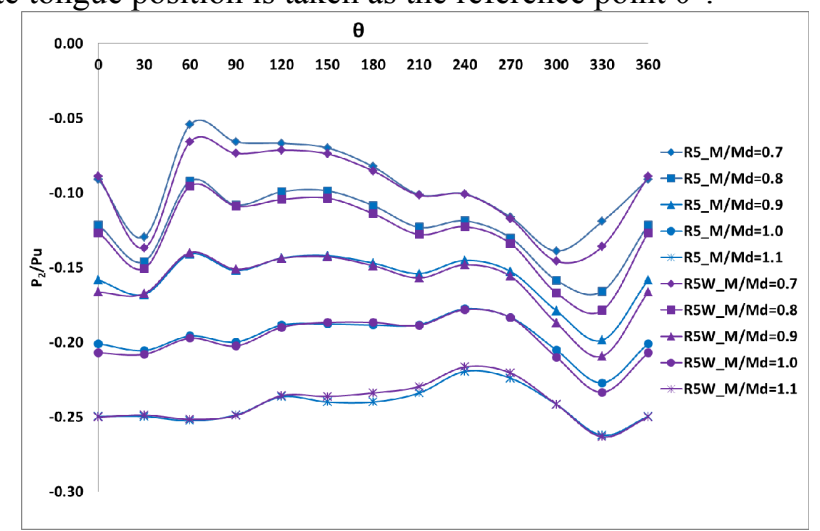

Fig. 9 Static pressure variation at inlet to impeller for R5 volute

The average static pressure at all mass flow rates at inlet are increased in the presence of clearance gap compared to the case with zero clearance. The increment closer to the tongue is much smaller when compared to the other positions. The distribution obtained for the R4 volute has more flow disturbances when compared to R5. This is because; the static pressure is the driving force. For the return flow, the static pressure is increasing along the volute circumferential. The distribution of static pressure for R4 and R5 along the periphery of the volute is not the same.

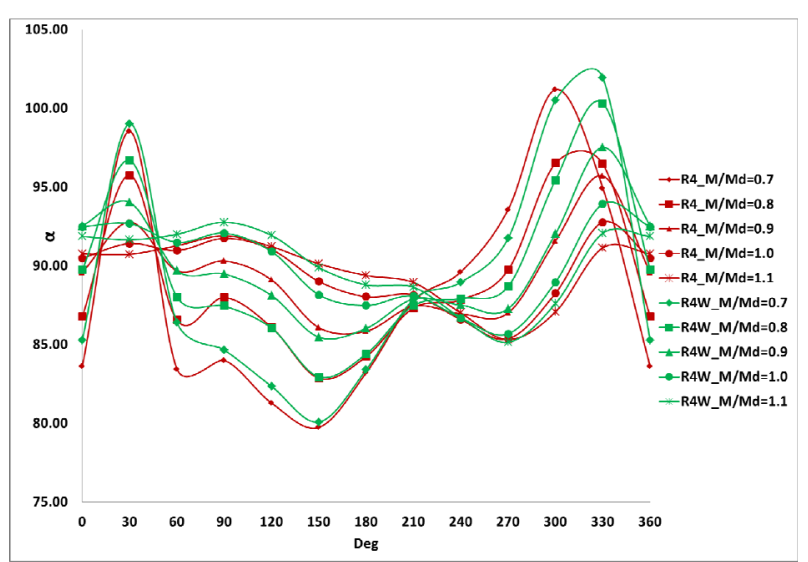

Fig. 10 Velocity flow angle at inlet to impeller for R4 volute

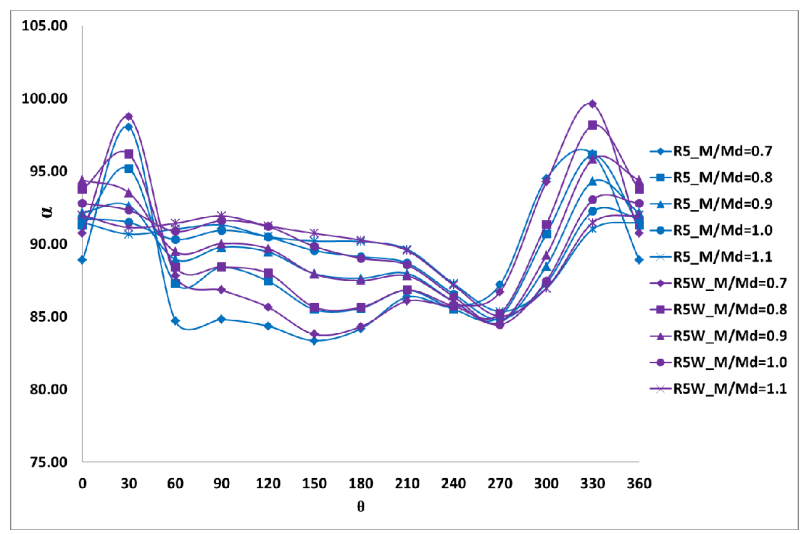

Fig. 11 Velocity flow angle at inlet to impeller for R5 volute

Another impact of the flow coming from the clearance is that it creates a cross flow to the inlet flow entering the impeller. In Fig. 11, the flow angle with respect to the circumference of the impeller is plotted as function of the angular position. There are no guide vanes in the present setup. The flow is expected to enter radially at $90^{\circ}$, however this is not the case due to the variation in pressure at the outlet of the impeller across the volute and also due to the off design condition. The change in the flow angle in the presence of clearance depicts an additional change leading to change in the loss and specific work produced by impeller.

\subsection{Volute Performance}

It is observed that when the clearance gap is zero, higher pressure rise in the impeller is observed. However, the fan performance is seen to vary from positive to negative. Hence such a scenario is possible only if the volute performance varies with the clearance. 
Loss co-efficient $(\omega)$, is defined as the ratio between the total pressure losses in volute to the dynamic pressure at the impeller exit.

$$
\omega=\mathrm{P}_{03}-\mathrm{P}_{04} / \mathrm{P}_{03}-\mathrm{P}_{3}
$$

Pressure recovery co efficient $\left(\mathrm{C}_{\mathrm{p}}\right)$ is defined as the ratio between the static pressure recovered in the volute to the dynamic pressure at the impeller exit.

$$
\mathrm{C}_{\mathrm{p}}=\mathrm{P}_{4}-\mathrm{P}_{3} / \mathrm{P}_{03}-\mathrm{P}_{3}
$$

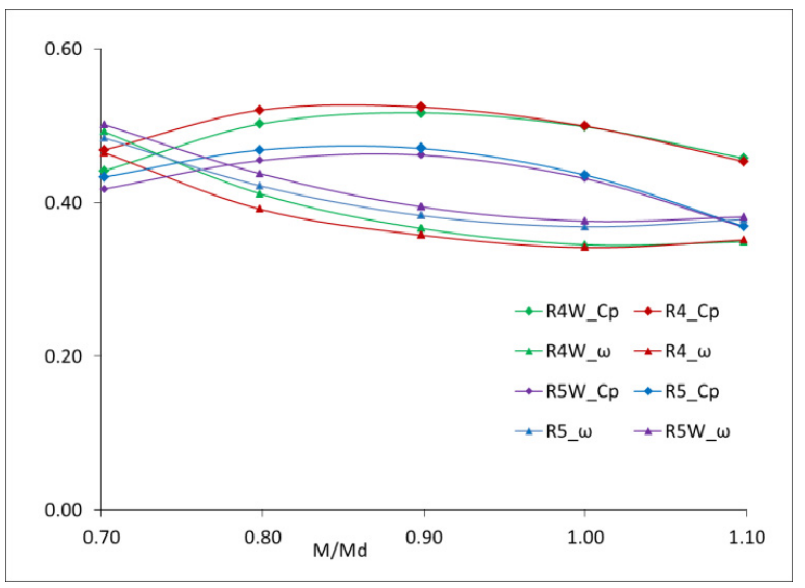

Fig. 12 Volute loss coefficients

In Fig. 12 the pressure recovery and the loss coefficients are plotted. It can be seen that the loss coefficients for zero clearance case tends to increase with decreasing mass flow rates and this is seen to occur even though the total pressure entering the volute is higher. This is due to the higher loss encountered in zero clearance case which in turn leads to a lower pressure rise across the fan at lower mass flow rates. At higher mass flow rates the loss coefficient is same for both cases. It can also be seen that there is an overall higher pressure rise at the outlet for zero clearance case. The difference in loss coefficient between zero and clearance is more for R4 volute when compared to the R5 volute. The pressure recovery co-efficient is high in the presence of clearance and it increases for lower mass flow rates. This indicates that clearance has a positive effect on the volute for all cases.

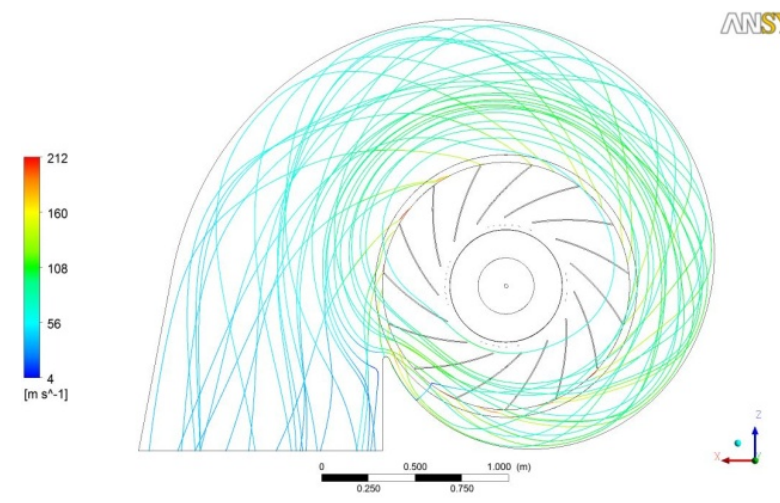

Fig. 13 Stream line inside volute at $\mathrm{M} / \mathrm{Md}=0.7$ for $\mathrm{R} 4$ with clearance

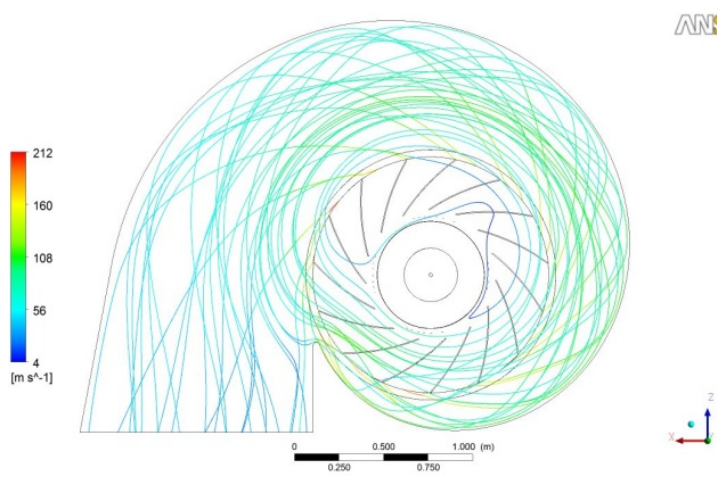

Fig. 14 Stream line inside volute at $\mathrm{M} / \mathrm{Md}=0.7$ for $\mathrm{R} 4$ without clearance

In order to qualitatively understand the loss mechanism in the volute, the stream lines are plotted from the impeller outlet. From the Figs 13 and 14, it is observed that the flow inside the volute for in the clearance case follows a logarithmic spiral path when compared to zero clearance case. Even though the velocity is almost same for both the cases, due to the presence of some recirculation and mixing, zero clearance case displays higher loss values when compared to the with clearance case. 

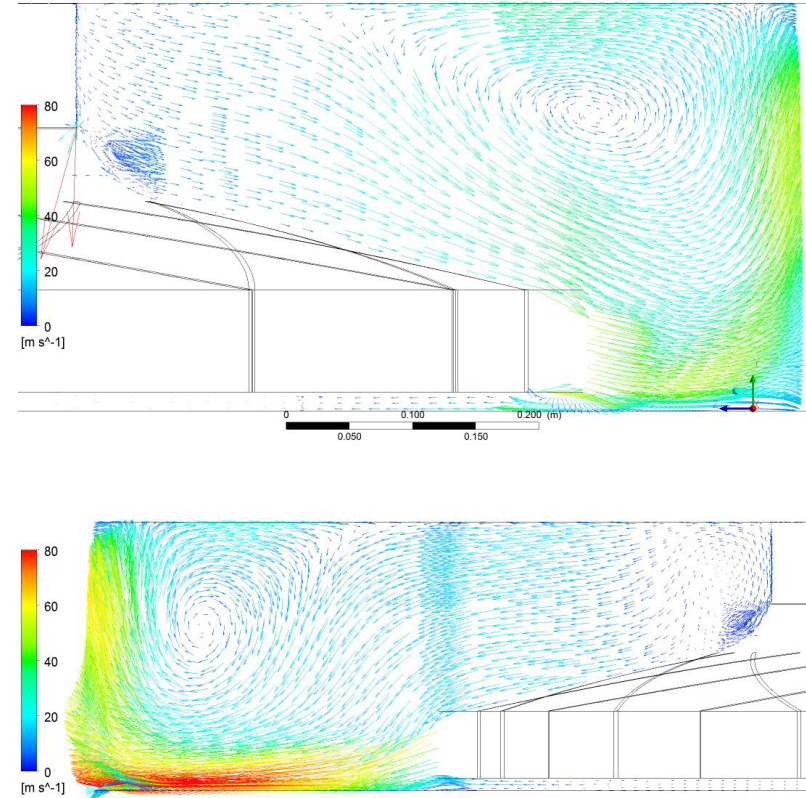

ANSSYS

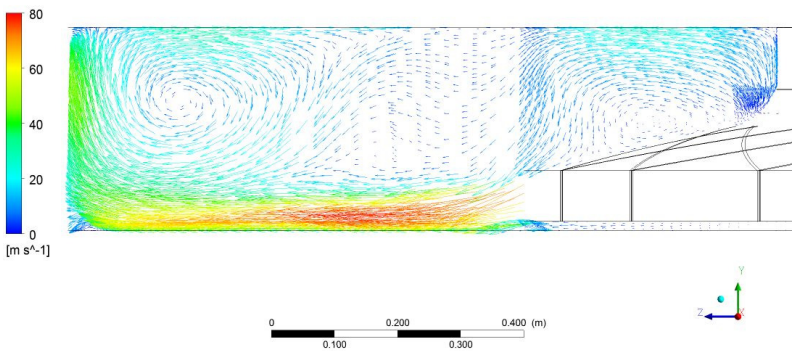

Nansys
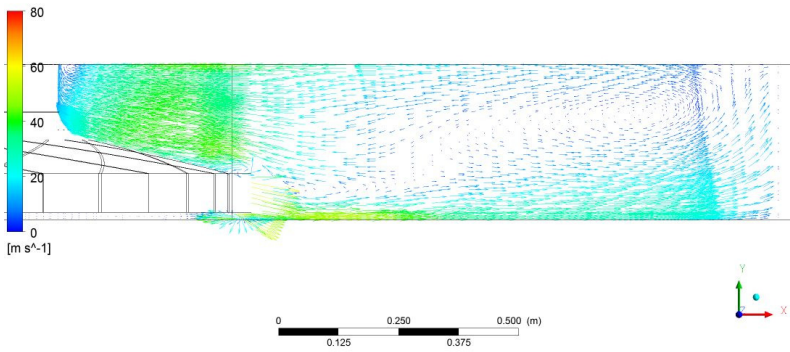

$\stackrel{\lfloor\cdot}{\longrightarrow}$

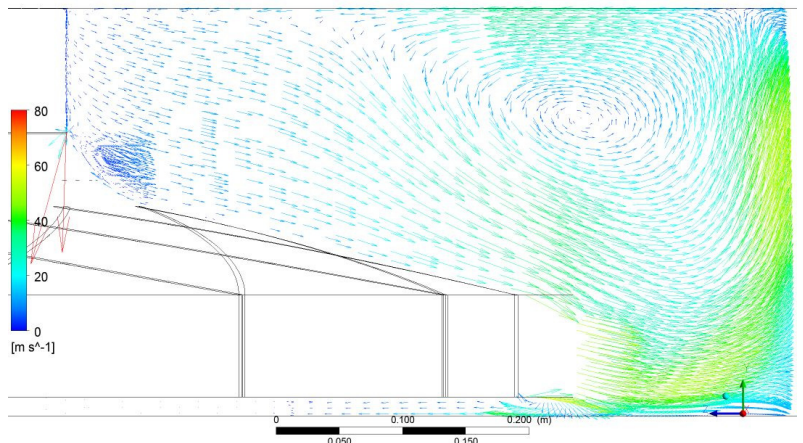

(a)

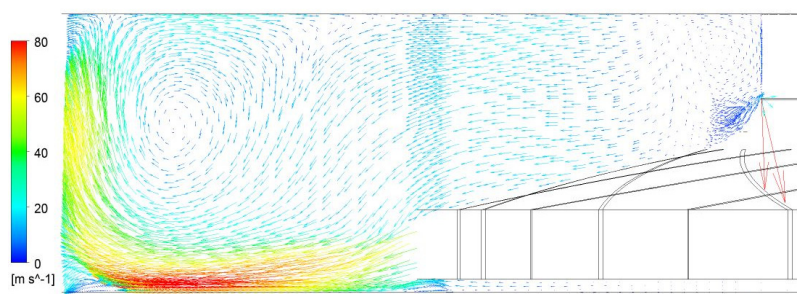

(b)

NNSYS

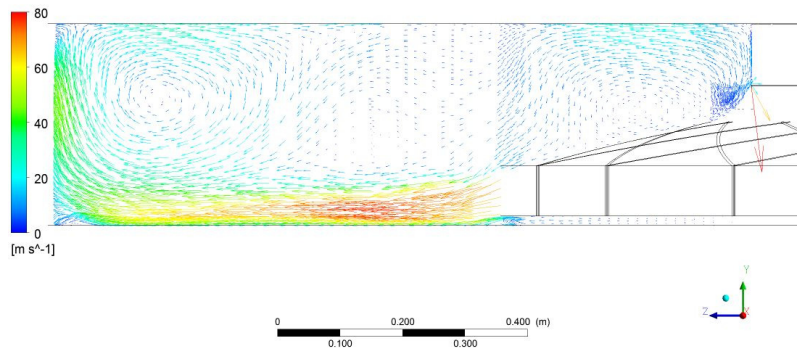

(c)

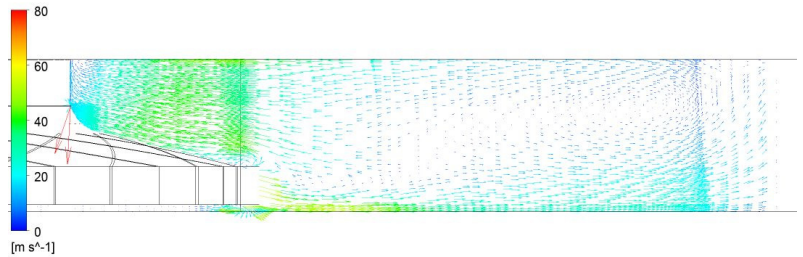

(d)

Fig. 15 Velocity vector at (a) $\theta=30^{\circ}$ (b) $\theta=120^{\circ}$ (c) $\theta=210^{\circ}$ (d) $\theta=330^{\circ}$ for $\mathrm{R} 4$ at $\mathrm{M} / \mathrm{M}_{\mathrm{d}}=0.7$

Left side depicts the without clearance results and the Right side is the with clearance results.

The velocity inside the volute is split into two components 1) through flow component (the velocity perpendicular to cross section of volute) 2) cross flow component (the velocity parallel to cross section of volute). Cross flow velocity is one of the main contributors to the loss in the volute. In Fig. 15 the velocity vector of the cross flow component is plotted for R4 volute at a mass flow rate of $\mathrm{M} / \mathrm{M}_{\mathrm{d}}=0.7$. We can see that the swirl center of the vectors for both cases are almost the same. The highest velocity is at the clearance where the flow is entering the impeller. The change in the vectors close to the clearance is very small. However the magnitude of the velocity vector in zero clearance case is marginally more. This is the reason for the higher loss in the volute. The loss in the volute is not produced because of the clearance but the clearance flow causes a disturbance at the exit of the impeller. 


\section{Conclusions}

The effect of clearance between the suction duct and impeller inlet in a centrifugal fan with parallel wall volute is studied in the present investigations. The clearance produces a positive effect on the pressure rise and the efficiency at lower mass flow rates when compared to the design mass flow rate conditions and negative effect at higher mass flow rates. Even though zero clearance case produces a higher pressure rise and efficiency in the impeller for all cases; due to the variation in the volute loss, the stage performance does not mimic a similar trend. The width ratio is observed to affect the results; higher the width ratio, smaller is the influence of clearance.

$\begin{array}{ll}\text { R4 } & \text { Width ratio } 4 \text { volute } \\ \text { R4 } & \text { Width ratio 5 volute } \\ \text { R4W } & \text { Width ratio 4 volute without clearance } \\ \text { R5W } & \text { Width ratio 5 volute without clearance } \\ \theta & \text { Angular position with respect to tongue } \\ \mathrm{M} & \text { Mass flow rate } \\ \mathrm{P} & \text { Static Pressure } \\ \mathrm{P}_{0} & \text { Total pressure } \\ \mathrm{Pu} & (1 / 2) * \rho * \mathrm{U}^{2} \\ \mathrm{U}_{2} & \text { Blade tip velocity } \\ \rho & \text { Density }\end{array}$

\section{Nomenclature}

$\begin{array}{ll}\mathrm{B}_{1} & \text { Impeller exit width } \\ \mathrm{B}_{2} & \text { Volute width } \\ \mathrm{M}_{\mathrm{d}} & \text { Design mass flow }\end{array}$

Subscripts

$\begin{array}{ll}1 & \text { Suction duct Inlet } \\ 2 & \text { Impeller Inlet } \\ 3 & \text { Exit of Impeller } \\ 4 & \text { Exit of volute }\end{array}$

\section{References}

[1] Y. T. Lee, 2010, "Impact of Fan Gap Flow on the Centrifugal Impeller Aerodynamics," Journal of Fluids Engineering 132.

[2] E. Ayder, T. Elholm, R.A. van den Braembussche, 1992, "The numerical investigation on the turbulent heat transfer," Journal of Turbomachinery 114: 366-372.

[3] R.A. van den Braembussche, K. Hillewaert, 1999, "Numerical Simulation of impeller - Volute Interaction in Centrifugal Compressors," Journal of Turbomachinery 121: 603-608.

[4] Gu. Fahua, 2001, “A Numerical Investigation on the Volute/Diffuser Interaction Due to the Axial Distortion at the Impeller Exit," Journal of Fluids Engineering 123: 475-483.

[5] Xiaominu Liu, Qun Dang, Xi. Guang, 2008, "Performance improvement of centrifugal fan by using CFD," Engineering application of computational Fluid Mechanics 2:130-140.

[6] Y. Kim, A. Engeda1, R. Aungier, 2001, "The influence of inlet flow distortion on the performance of a centrifugal compressor and development of an improved inlet using numerical simulation," Proc Instn Mech Engrs 215:323-338. 